\title{
Regímenes internacionales o anarquía internacional
}

Robert O. Keohane, After hegemony: Cooperation and Discord in the World Political Economy. Princeton University Press, 1984.

John Gerard Ruggie (ed.), The Antimonies of Interdependendence: National Welfare and International Division of Labor. Columbia University Press, 1983.

Stephen D. Krasner (ed.), International Regimens. Cornel University Press, 1983.

La teoría clásica de las relaciones internacionales ha dominado este campo de estudio durante el presente siglo con diversos altibajos. Salió triunfante de su confrontación con los idealistas al término de la primera guerra mundial y con los "behavioralistas", después de la segunda, y hasta ahora ha resistido el embate de Ios modernos "transnacionalistas" -particularmente a través de los neoconservadores que encontraron su paradigma en la primera administración de Reagan y tal vez encontrarán en la segunda su epitafio. Sus principales cultivadores han constituido las figuras cimeras en la disciplina: Aron, Mongerthau, Tucker, Waltz y muchos otros. Ellos describían el sistema internacional como una inestable y débil sociedad de estados cuya condición natural era la guerra y cuyo elemento morigerador era la disuación causada por la acumulación de recursos de poder. Hadley Bull describió este estado de cosas en una de sus ulltimas obras: The anarchical society: a Study of Order in World Politics.

A partir de los años setenta el bastión de la sabiduría convencional en el campo de las relaciones internacionales comenzó a sufrir el ataque de una nueva escuela que, bajo el impacto de la transformación experimentada por la realidad contemporánea, empezó a destacar sucesivamente nuevos rasgos como el papel de los actores no gubernamentales, la emergencia de una compleja red de relaciones transnacionales, el fortalecimiento de los vínculos de interdependencia entre las distintas naciones y el empleo de regimenes in- 
ternacionales para regular procesos globales mediante conocimientos y métodos técnicamente más sofisticados que el mero uso de la fuerza.

El último libro de Robert O. Keohane, que provocó estos comentarios, es una de las expresiones recientes de mayor importancia dentro de esta tendencia. Prosiguiendo por otros derroteros en la dirección abierta por Richard Cooper en 1968 con su libro The economics of interdependience, Keohane y Nye editaron en 1973 el influyente libro Transnational Relations and World Politics. A este trabajo, fuertemente sesgado hacia el rol de Ios actores transnacionales en la política mundial, siguió en 1967 otra obra preparada por los mismos autores -Power and Intendependence: World Politics in Transition-que comenzó a trasladar el énfasis desde los actores hacia los procesos transnacionales en que éstos participan y formalizó mucho más las categorías de análisis utilizadas para examinar estos fenómenos. Después de varios trabajos y aportes nuevos, en su último libro Keohane se centra en la función que desempeñan los regímenes internacionales desde el punto de vista de la administración de la interdependencia, definiéndolos -en forma por lo demás cambiante- como un conjunto de principios, reglas y procedimientos aceptados y puestos en práctica por un conjunto de actores internacionales para regular su participación en determinadas áreas o procesos y compatibilizar sus intereses.

La tesis central de Keohane, a lo largo de un análisis complejo, parece ser la de que la aceptación de las relaciones de interdependencia entre las distintas sociedades nacionales y la aplicación de regímenes internacionales para manejar dichas vinculaciones podría constituir una alternativa viable a la anarquía internacional, por una parte, o la imposición de un orden hegemónico, por la otra. El otro nombre para un mundo interdependiente, regulado por estos regímenes, es cooperación. Keohane destaca en su nuevo libro este concepto como contrapuesto al predominio de la discordia o el conflicto que ha caracterizado por regla general las relaciones entre los estados. Si ahora Keohane usa el concepto de cooperación porque está más interesado que en sus trabajos anteriores en el funcionamiento de la economía política, como se advierte en esta obra, o si sitúa su análisis en este último campo para dar pertinencia al concepto de cooperación (tomado del campo de la cooperación económica), es algo que no queda claro en el análisis. No es de extrañar que en una tarea ambiciosa como la que ha emprendido el autor, desde hace años se acumulen y utilicen paralelamente diversos conceptos que con frecuencia se superponen entre si: sistema internacional, interdependencia, economía politica internacional, instituciones y regímenes internacionales, entre otros.

Pese a estas limitaciones, propias de la amplitud de este es- 
tudio, el libro deja un saldo de avances valiosos. Uno, es el mismo intento de situar el análisis en el campo de la economía política internacional y por definir mejor sus parámetros. A diferencia de América Latina, donde la economía y la política han estado muy separadas en su desarrollo académico, existe en Gran Bretaña, en Europa continental y en los Estados Unidos una sólida tradición que vincula ambas disciplinas dentro del marco de la economía política. Recientemente se creó una sección sobre este tema dentro de la International Studies Association, lo que dio lugar a un primer libro que es interesante considerar en conexión con los que se mencionan en este comentario: An International Political EGonomy, editado en 1985 por W. Ladd Hollist y F. Lamond Tullis. Otro avance consiste en una visión más clara de las relaciones internacionales, no como un teatro donde las naciones compiten por el poder, sino como un sistema dentro del cual sus diversas partes deben articularse para desempeñar un conjunto de funciones. La adopción de regímenes internacionales para asegurar su cumplimiento maximizando la cooperación y minimizando el conflicto, yà sea en el caso del comercio de algunos productos básicos (como por ejemplo el café), la energía, los recursos del mar o el sistema monetario internacional, es un paso que naturalmente prolonga los anteriores. Otro aporte de esta obra consiste en atenerse en forma bastante rigurosa a una visión sistemática de las relaciones internacionales.

El libro editado por el profesor de Stanford, Stephen Krasner, constituye otra contribución importante al análisis de este tema. Después que varios autores analizan el papel de los regímenes internacionales desde la perspectiva de Hugo Grotio, y de que Oran Young estudia el auge y caída de este tipo de arreglos a la luz de una visión histórica, el propio Robert Keohane examina la demanda que existe actualmente por regimenes internacionales y, Arthur Stein, los procesos de cooperación a que dan lugar estos regímenes en un mundo anárquico. A continuación se examinan algunos regmenes imperantes en el campo de la seguridad, el orden económico internacional, el financiamiento y el comercio, para concluir esta visión con un debate entre el editor y la profesora inglesa Susanne Strange, una prestigiosa cultivadora de la economía política en el campo internacional, que cree más en el profesionalismo y en la habilidad negociadora que en la existencia de regímenes para el manejo cotidiano de los problemas que ésta plantea.

John Ruggie, profesor de la Universidad de Columbia, hace una contribución al estudio de las relaciones de interdependencia, altamente complementaria de la problemática anterior. Su aporte está basado en estudios de casos. Después de una lúcida introducción que contribuye a esclarecer una serie de conceptos que ya han sido 
mencionado; diversos autores analizan el desarrollo asociativo de los Estados Unidos entre 1776 y 1860; la situación de los países europeos pequeños; la posición de Corea del Sur en la economía internacional; las limitaciones que enfrentó Venezuela para llevar adelante un desarrollo asociado al sistema internacional, y los lf́nites de una estrategia orientada hacia las exportaciones en el caso de Costa de Marfil. En una segunda parte se examinan las dificultades que surgen para avanzar en el camino de la interdependencia en algunos sectores claves como el de la tecnología, la seguridad internacional o en las estrategias de desarrollo de los paises pobres de Africa. Todos los casos analizados en la obra ponen de manifiesto el contrapunto que existe entre el establecimiento o la mantención de una estructura internacional y los procesos de cambio que constantemente la afectan, destacando así la necesidad de armonizar ese contrapunto mediante la adopción de regimenes internacionales destinados a regular hasta cierto punto el comportamiento de las distintas partes dentro de cada sector.

La necesidad de desarrollar una polf́tica internacional más sectorializada en un mundo cada vez más interdependiente y tecnológicamente más complejo, y la conveniencia de establecer regímenes internacionales para encuadrar esa política, son las principales conclusiones de los estudios que se acaban de comentar. Los que se contenten con seguir viviendo en un estado de anarquía internacional cosecharán más competencia por el poder, más intertidumbre y más violencia. 